\title{
Triggered Star Formation: Rotation, Magnetic Fields and Outflows
}

\author{
A. Frank ${ }^{*, a}$, S. Li $^{\text {a }}$, E. G. Blackman ${ }^{\text {a }}$ \\ ${ }^{a}$ Department of Physics and Astronomy, University of Rochester, Rochester, NY \\ 14627-0171, USA
}

\begin{abstract}
Star formation can be triggered by compression from wind or supernova driven shock waves that sweep over molecular clouds. In a previous work we used Adaptive Mesh Refinement (AMR) simulation methods, including sink particles, to simulate the full collapse of a stable Bonnor-Ebert sphere subjected to a passing shock. We tracked the flow of cloud material after a star (a sink particle) had formed. For rotating clouds we observed the formation of disks which then interact with the post-shock flow. In this paper we take the next step forward in complexity, presenting first results of simulations that include a magnetized cloud. Our results show that after a disk is formed a collimated magnetocentrifugal outflow is launched. The outflow is bipolar but asymmetric, due to interactions with the shocked flow. We explore the influence of the outflows on the post-triggering collapse dynamics.
\end{abstract}

Key words: Shock wave phenomena, Star Formation, ISM, Jets and outflows

\section{Introduction}

Flow driven triggered star formation (TSF) occurs when a shock generated by distant supernova blast waves or stellar winds (wind blown bubbles) sweep over a stable cloud. In realistic environments, such flows likely impinge on heterogeneous regions within molecular clouds ([33], [21], [25], [2], [27]). While it is unclear if TSF accounts for a large fraction of the star formation rate within the galaxy, the concept has played an important role in discussions of the formation of our own solar system because it offers a natural way of injecting short lived radioactive isotopes (SLRI's) like ${ }^{26} \mathrm{Al}$ and ${ }^{60} \mathrm{Fe}$ into material which will then form planetary bodies. We note that ionization fronts sweeping over a cloud can also lead to triggering Krumholtz, et al [24] though we do not deal with those kinds of phenomena in this work.

\footnotetext{
* Corresponding author

Email address: afrank@pas.rochester.edu (A. Frank)
} 
Studies of TSF date back to the 1970s ([10], [32], [13]) with an emphasis on quantifying the ability of a blast wave or stellar wind to both trigger collapse in a stable cloud and inject processed material. Because of the complex nature of the resultant flows much of this work is based on numerical simulations ([4], [18], [35], [36]). Boss and collaborators in particular have provided an extensive series of studies ([5], [6], [8]) designed to articulate the shock conditions needed for successful triggering and mixing. In general, they find the higher the Mach number of the shock, the more difficult it is to trigger collapse. Faster shocks can shred and disperse the clump material before it has time to collapse. However faster shocks also allow better mixing by enhancing Rayleigh-Taylor instability growth rates [6]. Thus there is a relatively narrow window, in terms of shock Mach number, where both triggering and mixing can be achieved. It is worth noting that while $\mathrm{SN}$ shocks from nearby star forming regions can be expected to sweep over cores in some cases, triggering by HII regions may be more common ([15])

While these studies have revealed a great deal about flow-driven TSF, they have been restricted to the early stages of the resulting flow pattern. The full evolution leading to a collapsed object (a star) and its subsequent gravitational interaction with the surrounding gas remain to be studied. Part of the difficulty has been the numerical challenge of generating a sub-grid model for the collapsing region that adequately represent stars. Thus the mass accretion history of a star formed by triggering had not been quantified. The creation and long term evolution of accretion disks when rotation in present in the cloud has also not been explored in detail (see however Li et al. [28],citetBossea2014).

In addition the long term effect of magnetic fields in a flow driven TSF has yet to be explored van Loo et al [38]. In particular the presence of fields can alter the stability properties of the initial cloud, the dynamics of its collapse once it becomes gravitationally unstable and, perhaps, lead to the creation of magneto-centrifugal outflows if a disk is formed. The presence of outflow would be of particular interest because any material driven to escape velocity would be gas/dust that will not end up in the new planetary system. Thus the presence of fields, and the outflows they drive, might alter the delivery of mass (including processed elements) and hence change the mixing attributes of of TSF.

In this paper we present first results of a simulation study that includes magnetic fields. The presence of fields in molecular cloud cores, (the structures within which individual or binary stars are expected to form), have been confirmed through observations. These fields tend to be just below critical meaning they are not strong enough to support the core against its own gravity. Given the large compression factors expected when the cores collapse the fields can be expected to play a role in the dynamics of the system at smaller radii.

The structure of this first report of our ongoing campaign of simulations is as follows. In Section 2 we describe previous results. In section 3 we describe the numerical method and model. In Section 4 we report our results. Section 5 provides our conclusion. 


\section{Previous Results}

In [28] the parallel AMR code AstroBEAR2.0 ([14], [11]) was used to study the shock-induced triggering of a stable Bonnor-Ebert cloud following, for the first time, the long-term evolution of the flow after a star, numerically represented by a sink particle, has been formed. Cases with and without initial rotation of the BE sphere were studied. That work found that the TSF interaction proceeds in three phases. First the shock compresses the cloud enough to form a star at the core. Then cloud material gets ablated and unbound from the star. Finally some material remains bound to the star and continues to evolve as it is exposed to the post-shock flow. The study also found that the shock Mach number affects the asymptotic stellar mass more than the rotation: the higher the Mach number, the less the stellar mass at the end of the simulation. In the case of rotating clouds, bound disks we observed to form around the newly formed stars which were then exposed to the post-shock flow. The disk, however, were quite stable surviving wind erosion with total momentum injection of $0.05 M_{\odot} \mathrm{km} / \mathrm{s}$ given the parameters used. Mixing was found to be sensitive to mach number (as expected) with a lesser sensitivity to the presence and inclination of rotation.

\section{Numerical Model}

Our simulations utilize the AstroBEAR 2.0 code using a 3D computational grid. Details on AstroBEAR may be found in [14], [11]. While the code can treat multiple atomic, ionic and molecular species, in this work we use a single fluid assumption with tracers to track different types of material: cloud, initial ambient and post-shock. We also use an approximate isothermal assumption: $\gamma=1.0001$. At each step the gravitational potential is calculated using linear solver package HYPRE ([3]), which is then passed to the fluid equations solver ( MUSCL primitive method with TVD preserving Runge-Kutta temporal interpolation). For details of self-gravity in AstroBEAR see [23].

The formation of the star is treated numerically as the introduction of a sink particle. When the local fluid quantities satisfy a set of criteria ensuring collapse within a simulation cell, a particle of appropriate mass is created and subsequently treated as a point gravity source. The zero-dimensional particle, once formed, moves through the grid based on gravitational interactions with surrounding gas and other particles. In this paper, we form particles based on criteria proposed by [17], and then calculate the accretion of the particle based on the accretion scheme described in [26].

We begin with an initial marginally stable Bonnor-Ebert sphere as the triggering target The initial cloud setup is similar to [6], i.e a cloud with $M_{c}=1.1 M_{\odot}$, a radius of $R_{c}=0.058 p c$, a central density of $\rho_{c}=6.3 \times 10^{-19} \mathrm{~g} / \mathrm{cc}$ and edge density of $3.6 \times 10^{-20} \mathrm{~g} / \mathrm{cc}$. The cloud has a uniform interior temperature of $10 \mathrm{~K}$. The ambient medium is setup to satisfy the pressure balance at the cloud edge when the cloud is stationary. To characterize the importance of rotational energy in our simulations we use $K=\Omega \times t_{f f}$, where $t_{f f}$ is the 
free-fall time and $\Omega$ is the angular velocity. We assume $K=0.1$ for all the rotational cases presented in this paper ([1]). The magnetic field simulation assumes a purely poloidal field distribution that is entirely embedded within the $B E$ sphere. Thus field lines take on an topology resembling orange slices. The strength of the field is described by an average $\beta$ which our simulations is $\beta=12$. For more details on the field set-up readers should consult [29]. We note that with a contained magnetic field and initial rotation the $\mathrm{BE}$ cloud is no longer in equilibrium, but the expansion from the poloidal component and rotation are both small with no noticeable expansion during the simulation time scale.

We drive a Supernova blast wave "shell" over the BE sphere with mach number $M=1.5$, the shock speed is $5 \mathrm{~km} / \mathrm{s}$, shell width is $0.003 \mathrm{pc}$, shell density is $3 * 10^{-20} \mathrm{~g} / \mathrm{cc}$. After the shell, we continue to inject a "post-shock wind" with density $7.2 * 10^{-22} \mathrm{~g} / \mathrm{cc}$ for the duration of the simulation. See [28] for more details about the initial conditions.

\section{Results}

In figure 1 and 2 we present results from our simulation that begins with a rotating magnetized $\mathrm{BE}$ sphere. The figures show color maps of density with contours of magnetic pressure (left) and magnetic field lines with contours of density (right). Figure 1 show cuts aligned with the direction of propagation of the shock (the poloidal mid-plane). Figure 2 shows the perpendicular view, i.e. cuts made through the equator of the BE sphere. We note that the evolution of the non-rotating and non magnetized rotating cases have already been described in [28] and the reader should consult that work for more details.

At $t=0.285 \mathrm{Myr}$ the supernova shock has already swept over the BE sphere and initiated collapse. Figures 1 and 2 shows that a sink particle has already formed in at this time at the cloud center. Note in figure 1 (left) that the contours of field pressure reflect the compression of magnetic energy into the equatorial plane at small radii. Note, however, that both the density map (left) and density contours (right) in figure 1 show that a disk has not yet formed. Figure 2 (right) does show the initially poloidal fields being generally distorted by differential rotation, although only in the central most regions do we see the field being converted from poloidal to toroidal configurations. It is these regions that are causing the contours of field pressure to pinch in towards the equator in fig 1.

By $t=0.36$ Myr two important transitions have occurred. First a rotating disk has formed. Second, with the disk formation a bipolar outflow has also been launched above and below the inner disk surface. From the density map in figure 1 (b) it is clear that the lobes of the bipolar outflow are not symmetric. The jet/outflow launched upstream, into the post-shock wind, propagates faster and farther than the outflow launched downstream. We attribute this asymmetry to the higher average density downstream of the sink particle. This higher density occurs due to the the formation of a turbulent velocity/density field just downstream of the cloud. It also occurs due to the elongation of the cloud by the shock. Thus the downstream-oriented outflow must first propagate through 

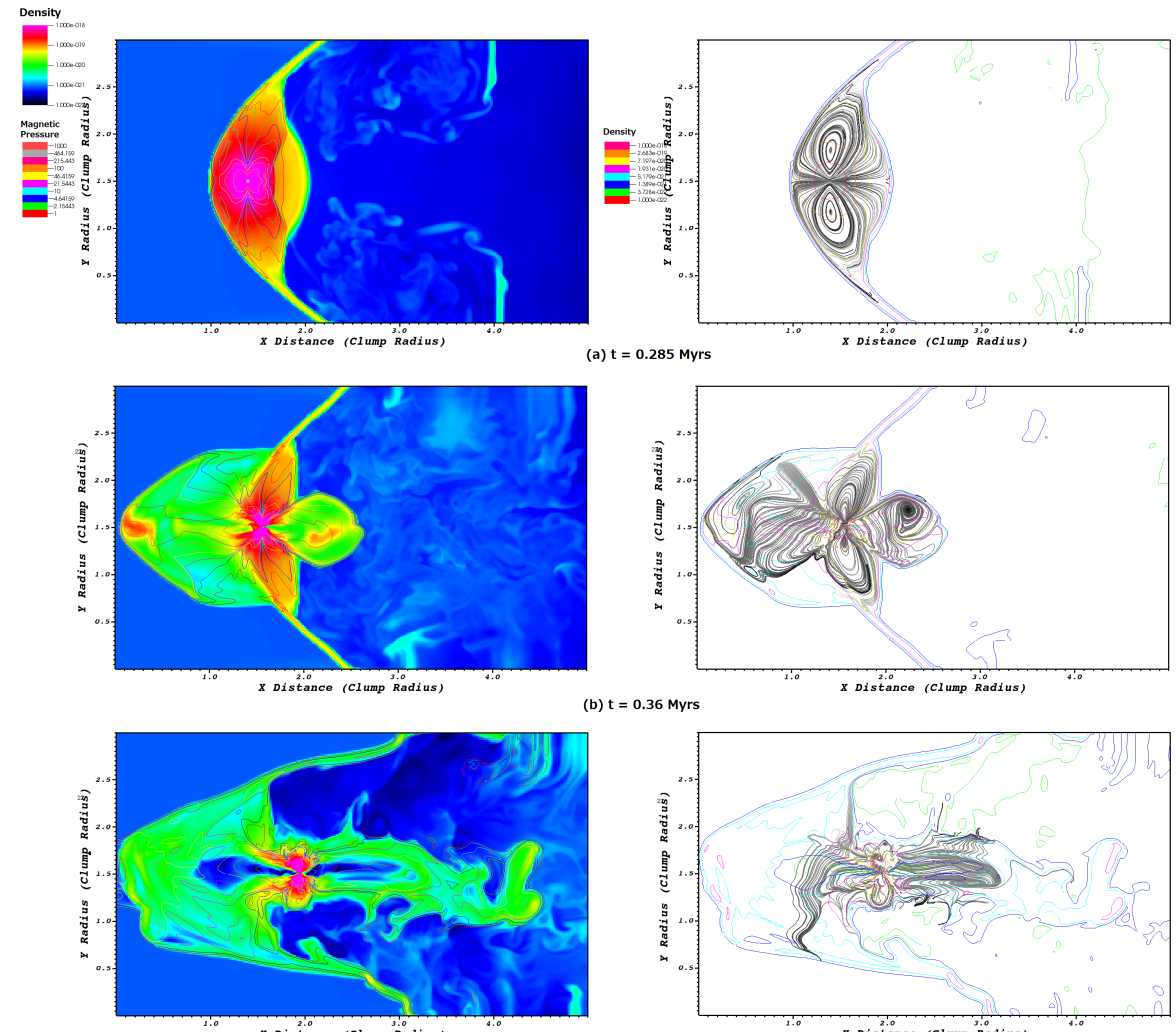

(b) $\mathrm{t}=0.36$ Myrs

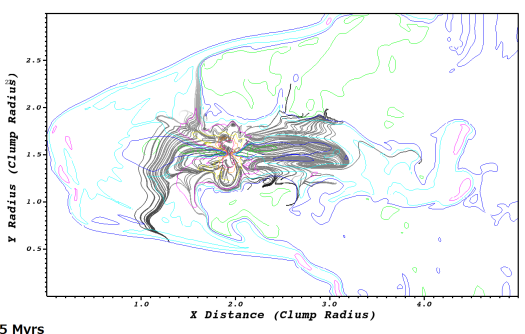

Figure 1: 2-D cuts of 3-D simulation of triggered star formation simulation using a magnetized rotating BE sphere as target. Axis of rotation and magnetic axis of poloidial field are parallel to the direction of propagation of incoming shock (shock travels left to right). Cuts are taken in the plane perpendicular to the BE sphere's equator. Left: Density map with magnetic pressure contours. Right: Magnetic field lines with density contours. 

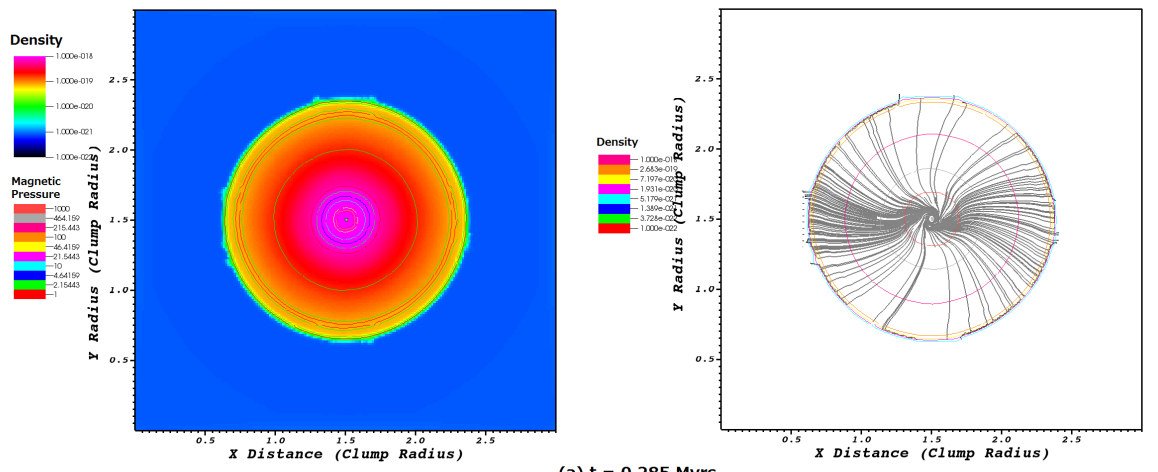

(a) $t=0.285$ Myrs
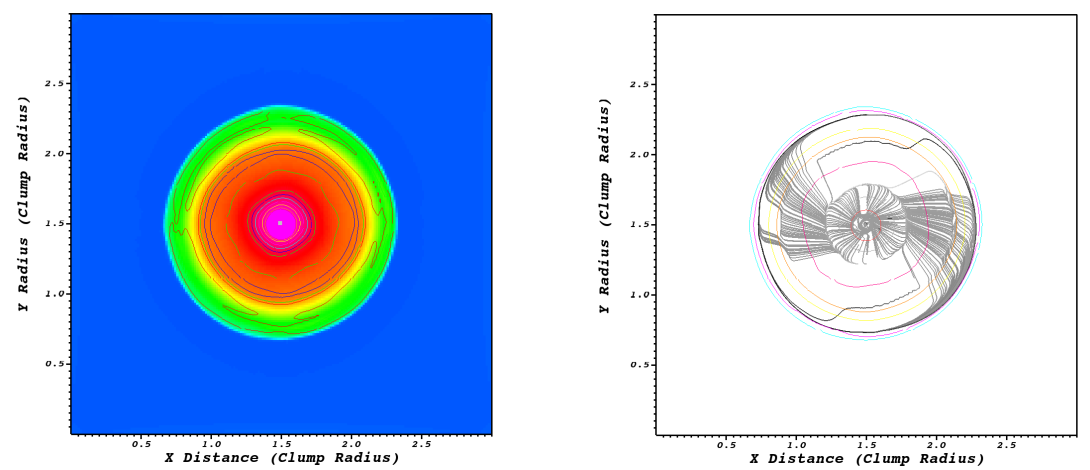

(b) $\mathrm{t}=0.36$ Myrs
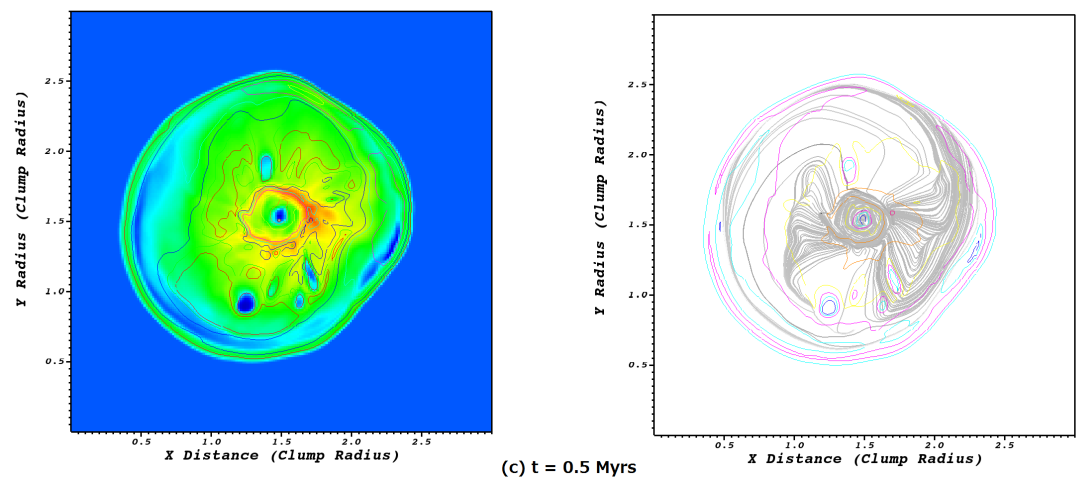

Figure 2: As in figure 1 with cuts taken in BE sphere's equatorial plane. Left: Density map with magnetic pressure contours. Right: Magnetic field lines with density contours. 
the dense cloud and the through a turbulent medium outside the cloud. The upstream propagating outflow on the other hand has less cloud material to pass through initially. The upstream propagating outflow does however interact with the post-shock wind and a well-defined bow shock is apparent at the head of the outflow system. Note that the field line map (which only shows poloidal projection) indicates a mostly toroidal geometry for the outflows at this time with at least two significant local perturbations/vortices. Turning the equatorial plane shown in fig2 we see in both the density map and field lines that at this time distinct concentration of density at smaller radii has occurred as inflowing the material collects into disk.

By $t=0.5$ Myr the collimated bipolar flow has significantly evolved (fig 1 bottom). Note in fig1 that by this time the downstream propagating outflow has completely broken free of the parent cloud exhibiting. It now shows considerable density inhomogeneities most likely due to the turbulent medium it has propagates through for some distance. The well-defined bow shock surrounding the upstream jet is still apparent. Note that in fig 1 the disk is now clearly apparent in the density map. The field lines seen in fig 2 also show a clear articulation of the disk structure in the region where poloidal field lines have been completely converted to toroidal geometry.

In Figure 3 we present volume render images of density at $t=0.36$ Myrs. The top image uses a higher "opacity" in the rendering and highlights the upstream outflow and the shock sweeping over the cloud. Note the structure of the upstream outflow (blue) with distinct knots forming at and near the /bow shock. The bottom image allows us to see inside the cloud. The disk is clearly apparent. The cavity driven by the downstream collimated outflow is also visible. Note the presence of denser material associated directly with outflowing inside the bulb-shaped cavity

\section{Final Stellar Mass}

A critical question to be addressed in studies of flow driven TSF is the final mass of the star formed by the triggered collapse. The use of sink particles allows the long term evolution of the collapsing system to be studied up to the point when the "star" appears to reach its asymptotic mass value $M_{m f}$. In figure 4 we show the evolution of sink particle mass for 3 cases: a non-rotating nonmagnetized cloud (red); a rotating non-magnetized cloud (black); the rotating magnetized cloud simulation discussed in this paper (blue). We note that the simulation shown in this paper is not calculated with the same resolution as the other two cases. Because the sink particle captures all gas that is inflowing towards the central regions, however, once collapse has been initiated we do not expect the resolution to influence these results to 1st order.

Figure 4 shows a clear trend with respect to final mass $M_{m f}$. The nonrotating non-magnetized, rotating non-magnetized and rotating magnetized cases produce, respectively $M_{m f} \sim 1.0,0.9,0.8 M_{\odot}$. As was discussed in [28] the decrease in mass between the non-rotating and rotating cases can be attributed to the creation of the accretion disk. Gas parcels which would have fallen into the 


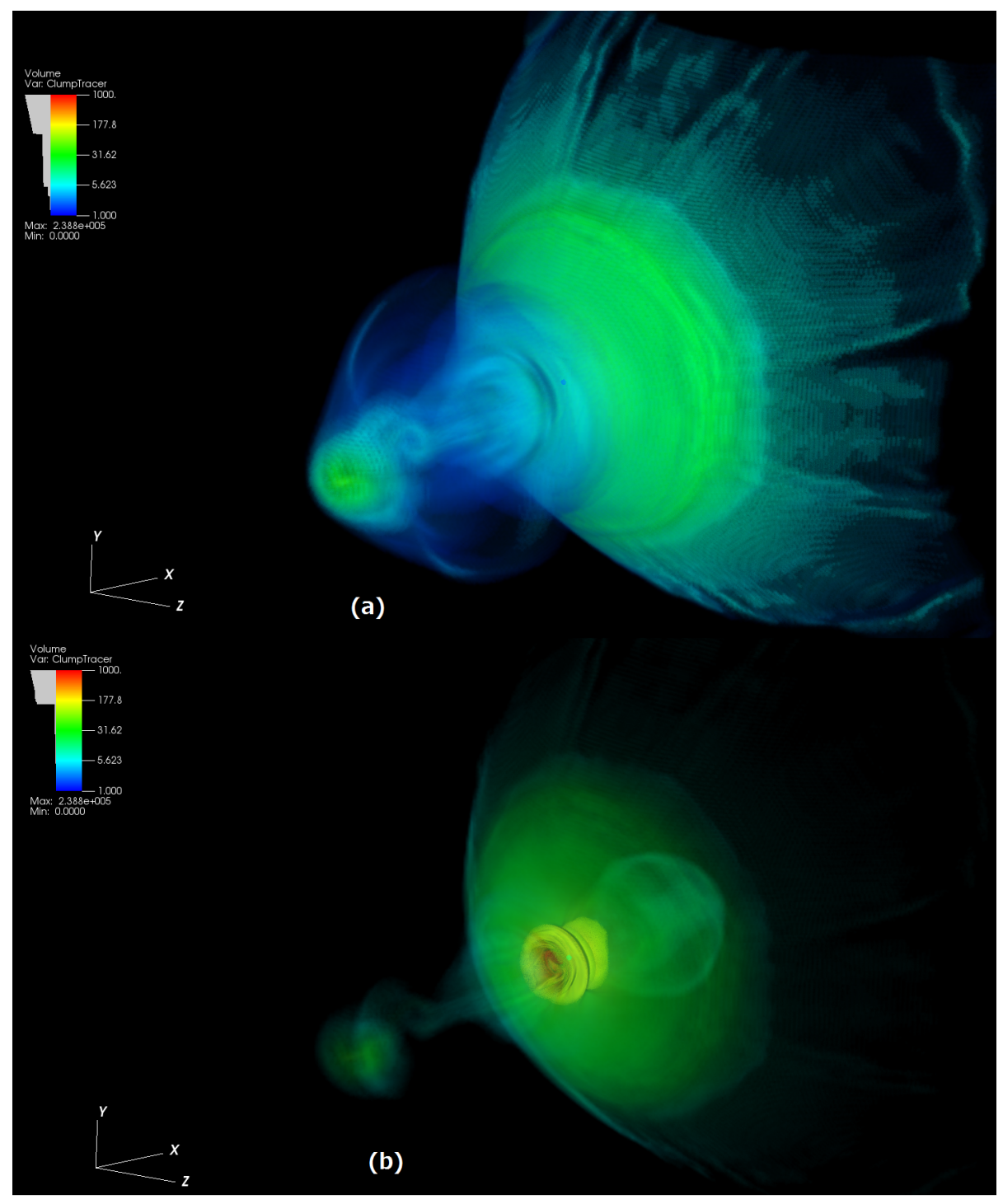

Figure 3: Two volume rendered images of the flow taken $t=0.36 \mathrm{My}$. The upper image has high "opacity" and highlights the shock over-running the cloud and the upstream propagating jet (relative to the direction of the incident shock) . The lower image has lower "opacity" and highlights the cavity driven into the cloud by downstream propagating jet. 


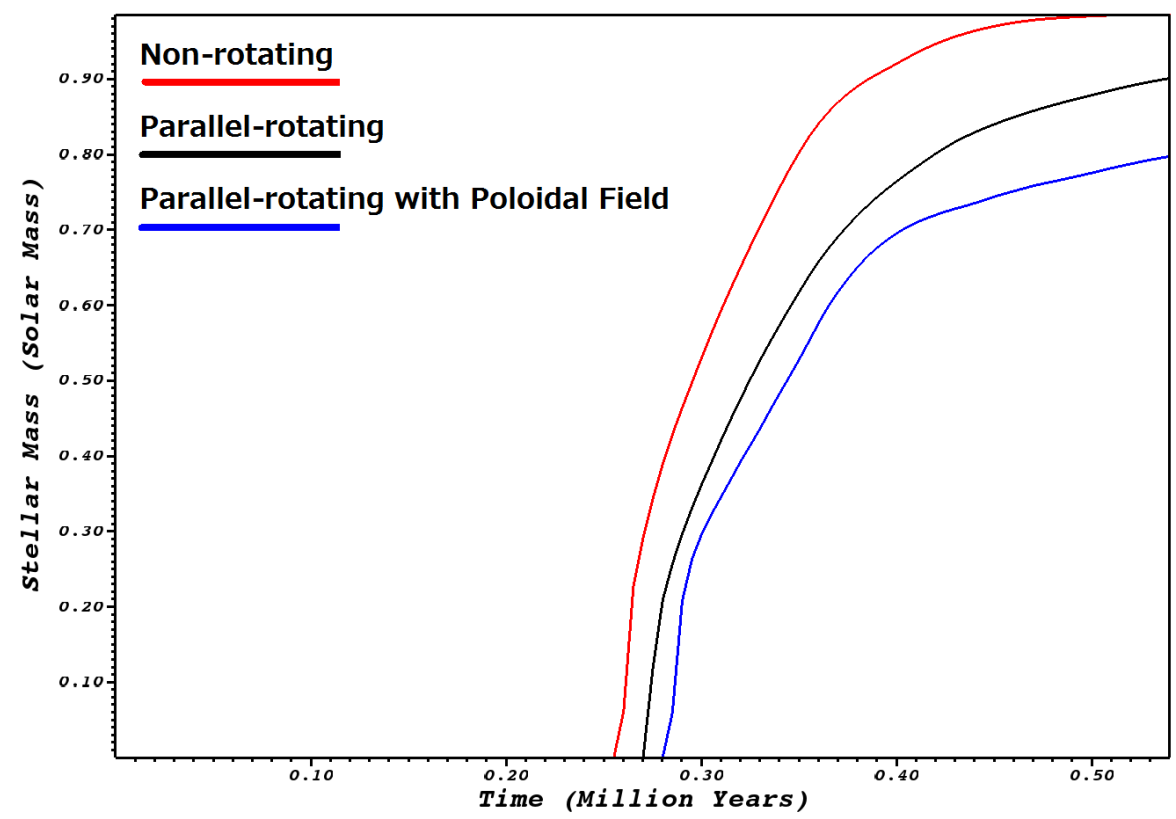

Figure 4: Evolution of stellar (sink particle) mass for three runs. Note the sequence in terms of final mass $M_{m f}$ achieved in the simulation. Non-rotating and non-magnetized > rotating and non-magnetized $>$ rotating and magnetized

sink particle are now in stable orbits within the disk. The addition of poloidal magnetic fields however, leads to the creation of outflows as we have seen. The gas in the outflow is driven to escape velocity and that material will not become part of the star. In addition, the outflow will sweep up in-falling cloud material. Thus the additional drop $M_{m f}$ with the addition of magnetic fields is consistent with the dynamics seen in the simulation images.

\section{Conclusions}

Star formation can be triggered by compression from a wind or a supernova driven shock wave that sweeps over a molecular clouds. In a previous work, [28], we used Adaptive Mesh Refinement simulation methods, including sink particles, to simulate the full collapse and subsequent evolution of a stable Bonnor-Ebert sphere subjected to a passing supernova shock. For rotating clouds we observed the formation of disks which then interact with the postshock flow.

In this paper we present the first results of simulations that include a magnetized cloud. Our results show that after a disk is formed, a collimated magnetocentrifugal outflow is launched. While the outflow is bipolar it is asymmetric due to interactions with the post-shock flow. We explored the outflows impact 
on the post-triggering collapse dynamics and found they appear to reduce the final stellar mass $M_{m f}$ by driving material in the disk to escape and sweeping up cloud material that is still in-falling.

This work represents a first report of a campaign of simulations designed to study MHD flow driven shock triggering. Future work will explore a broad range of initial conditions simulated at higher resolution. The discovery of magnetocentrifugal outflows in TSF flows is significant however because it represents an example of what might be termed multi-level feedback. The triggering event is itself a form of stellar feedback (on molecular cloud scales). The triggering leads to an outflow which drives its own feedback, now apparent on the star forming core scale.

Acknowledgements. This work used the Extreme Science and Engineering Discovery Environment (XSEDE), which is supported by National Science Foundation grant number OCI-1053575. The CIRC at the University of Rochester

provided computational resources. Financial support for this project was provided by the LLE at the University of Rochester, Space Telescope Science Institute grants HST-AR-11251.01-A and HST-AR-12128.01-A; by the National Science Foundation under award AST-0807363; by the Department of Energy under award de-sc0001063.

\section{References}

[1] Banerjee, R., Pudritz, R. E., Holmes, L., 2004, MNRAS, 355, 248

[2] Bonnell, I. A., Dobbs, C. L., Robitaille, T. P., Pringle, J. E., 2006, MNRAS, 365, 37

[3] Baker, A. H., Falgout R. D., Kolev, Tz. V., Yang U. M., 2012, in High Performance Scientific Computing: Algorithms and Applications, eds. Springer, Scaling hypre's Multigrid Solvers to 100,000 Cores, LLNL-JRNL-479591

[4] Boss., A. P., 1995, ApJ, 439, 224

[5] Boss, A. P., Ipatov, S. I., Keiser, S. A., Myhill, E. A., Vanhala, Harri A. T., 2008, ApJ, 686, 119

[6] Boss, A. P., Keiser, S. A., Ipatov, S. I.,Myhill, E. A., Vanhala, Harri A. T., 2010, ApJ, 708, 1268

[7] Boss, A. P., Keiser, S. A., 2010, ApJ, 717, 1

[8] Boss, A. P., Keiser, S. A., 2013, ApJ, 770, 51

[9] Boss, A. P., Keiser, S. A., 2014, ApJ, in press

[10] Cameron, A. G. W., Truran, J. W., 1977, Icarus, 30, 447 
[11] Carroll-Nellenback, Jonathan J., Shroyer, Brandon Frank, Adam, Ding, Chen, 2013, J. Comp. Phy., 236461

[12] Chevalier, R. A. 2000, ApJ, 538, 151

[13] Clayton, Donald D., Hartmann, Dieter H., Leising, Mark D., 1993, ApJ, 415,25

[14] Cunningham, A.J., Frank, A., Varnière, P., Mitran, S., Jones, T.W., 2009, ApJs, 182519

[15] Dale, J.E., Ngoumou, J., Ercolano, B., Bonnell, I.A. 2014. Monthly Notices of the Royal Astronomical Society 442, 694-712.

[16] Dhanoa, H., Mackey, J., Yates, J., 2014, http://arxiv.org/abs/1402.1103

[17] Federrath, C., Banerjee, R., Clark, P. C., Klessen, R. S., 2010, ApJ, 713, 269

[18] Foster, P. N., Boss, A. P., 1996, ApJ, 468, 784

[19] Gritschneder, M., Lin, D. N. C., Murray, S. D., Yin, Q. Z., Gong, M. N., 2012, ApJ, 745, 22

[20] Gong, Hao, Ostriker, Eve C., 2013, ApJs, 204, 8

[21] Hillenbrand, L.A., 1997, AJ, 113, 1733

[22] Jones, T. W., Ryu, Dongsu, Tregillis, I. L., 1996, ApJ, 473, 365

[23] Kaminski, E., Frank, A., Carroll-Nellenback, Jonathan J., Myers, P., submitted to ApJ, http://arxiv.org/abs/1401.5064

[24] Krumholtz, M., et al, 2014, Star cluster formation and feedback, accepted for publication as a chapter in Protostars and Planets VI, University of Arizona Press (2014), eds. H. Beuther, R. Klessen, C. Dullemond, Th. Henning

[25] Kothes, R., Uyaniker, B., Pineault, S., 2001, ApJ, 560, 236

[26] Krumholz, M. R., McKee, C. F., Klein, R. I., 2004, ApJ, 611, 399

[27] Leao, M. R. M., de Gouveia Dal Pino, E. M., Falceta-Goncalves, D., Melioli, C.; Geraissate, F. G., 2009, MNRAS, 394, 157

[28] Li, S., Frank, A., \& Blackman, E. 2014, MNRAS in press, arXiv:1404.5924

[29] Li, S., Frank, A., \& Blackman, E. G. 2013, ApJ, 774, 133

[30] Mac Low, M., McKee, C.F., Klein, R.I., Stone, J.M., Norman, M.L., 1994, ApJ, 433, 757

[31] Ouellette, N., Desch, S. J., Hester, J. J., 2007, ApJ, 662, 1268 
[32] Reynolds, R. T., Cassen, P. M., 1979, Geo. Rev. Lett., 6, 121

[33] Roberts., W. W., 1969, ApJ, 158, 123

[34] Walch, S., Whitworth, A. P., Bisbas, T. G., Wnsch, R., Hubber, D. A., 2013, MNRAS, 435, 917

[35] Vanhala, Harri A. T., Cameron, A. G. W., 1998, ApJ, 508, 291

[36] Vanhala, Harri A. T., Boss, A. P., 2002 ApJ 575, 1144

[37] Vaidya, B., Hartquist, T. W., Falle, S. A. E. G., 2013, MNRAS, 433, 1258

[38] van Loo, S., Falle, S. A. E. G., Hartquist, T. W., Moore, T. J. T., 2007, AA, 471, 213 